\title{
Secukinumab: Rapid Efficacy in Psoriasis After Primary Failure With Ustekinumab and Adalimumab
}

\author{
Aline Lissa Okita ${ }^{1}$, Tatiane Benini ${ }^{1}$, Denise Reis Longhi ${ }^{1}$
}

1 Department of Dermatology, Universidade de Mogi das Cruzes, São Paulo, Brazil

Key words: severe psoriasis, biologics, secukinumab, adalimumab, ustekinumab

Citation: Okita AL, Benini T, Reis Longhi D. Secukinumab: rapid efficacy in psoriasis after primary failure with ustekinumab and adalimumab. Dermatol Pract Concept. 2020;10(4):e2020102. DOI: https://doi.org/10.5826/dpc.1004a102

Accepted: May 28, 2020; Published: October 26, 2020

Copyright: (2020 Okita et al. This is an open-access article distributed under the terms of the Creative Commons Attribution License BYNC-4.0, which permits unrestricted noncommercial use, distribution, and reproduction in any medium, provided the original author and source are credited.

Funding: None.

Competing interests: The authors have no conflicts of interest to disclose.

Authorship: All authors have contributed significantly to this publication.

Corresponding author: Aline Lissa Okita, MD, Department of Dermatology from Universidade de Mogi das Cruzes, Ave Dr Cândido X de Almeida e Souza, 200, São Paulo, Brazil. Email: alinelissaokita@gmail.com

\section{Introduction}

Several guidelines recommend phototherapy, systemic agents, or biologic therapy for the treatment of moderate-to-severe psoriasis. Sequential use of more than 1 biologic has become more common due to primary or secondary failure with this type of treatment and an increase in the number of drugs available. However, the order of usage of these drugs is still speculative. We report about a patient with severe psoriasis with primary failure to respond after treatment with ustekinumab and adalimumab who achieved psoriasis area and severity index (PASI) 100 score with secukinumab in 8 weeks.

\section{Case Presentation}

A 47-year-old woman presented with a 30-year history of psoriasis vulgaris without psoriatic arthritis or other comorbidities. She was previously treated with methotrexate and PUVA but had hepatotoxicity and minimal response with UVB narrowband.

At first visit, the patient revealed PASI 23.7, dermatology life quality index (DLQI) 30, body surface area (BSA) 30 , and body weight $80 \mathrm{~kg}$ (Figure 1) and was subsequently

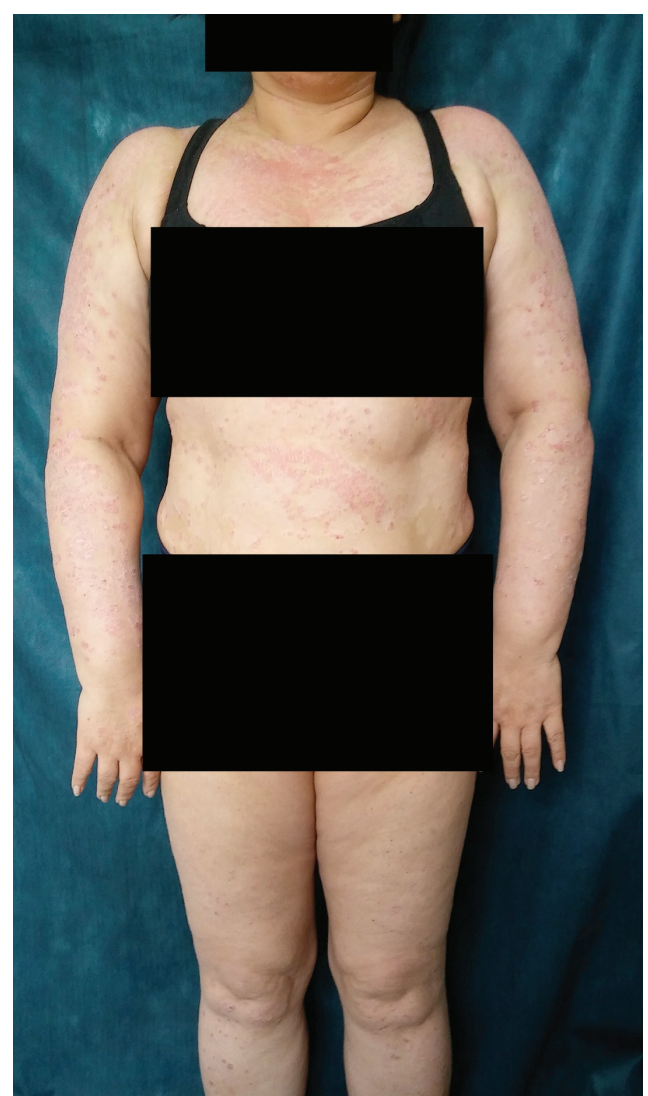

Figure 1. Clinical presentation prior to ustekinumab treatment. 

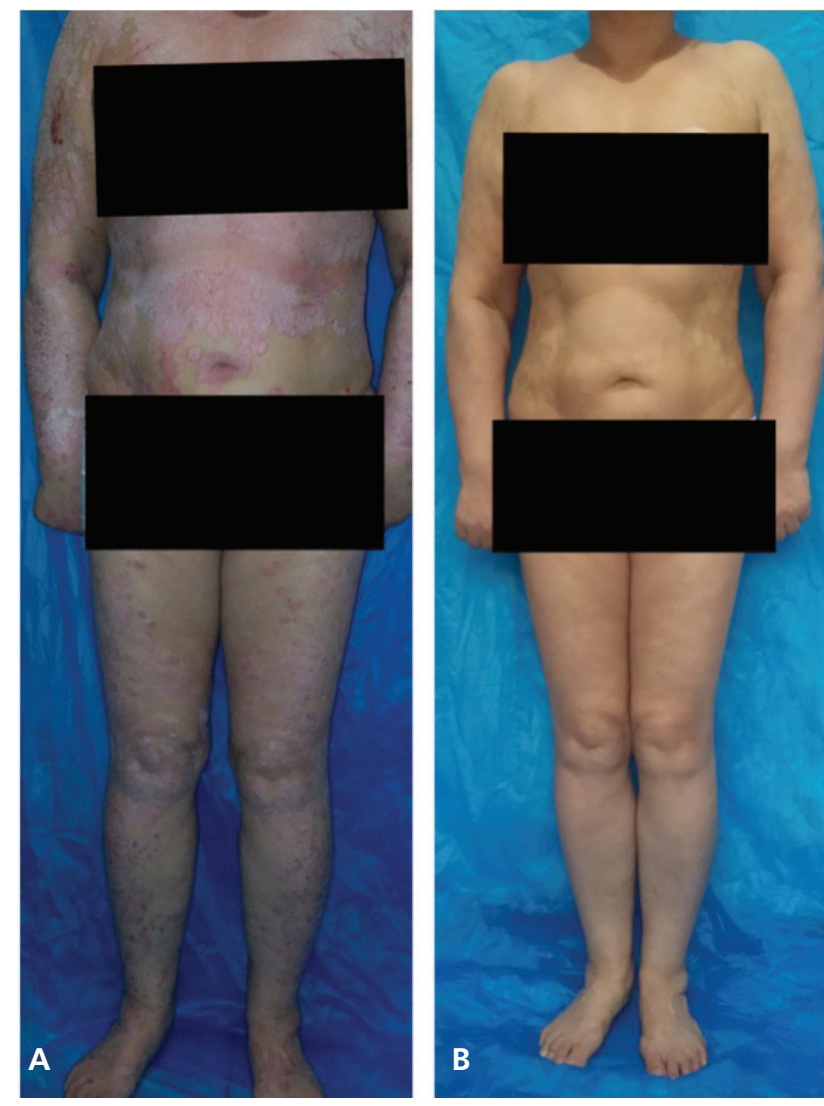

Figure 2. Clinical presentation. (A) After adalimumab treatment. (B) After 5 weeks of secukinumab treatment.

treated with ustekinumab $45 \mathrm{mg}$ for 6 months with no response. Then she received adalimumab, but symptoms worsened with an increased number of lesions and intense itching (PASI 33.6, DLQI 30, BSA 78) (Figures 2A and 3A). After that, she received secukinumab $300 \mathrm{mg}$ at weeks $0,1,2$ and 3, 4 achieving PASI 75 at week 5 (Figures 2B and 3B) and PASI 100 and DLQI 0 at week 8.

\section{Conclusions}

Studies on secukinumab have demonstrated rapid and high efficacy in the treatment of psoriasis, and bio-naive patients achieved higher scores, PASI 90, than those previously exposed to treatment with biologics.

However, many authors reported successful treatment with secukinumab after biological exposure. In one series of 6 patients, patients exhibited efficacy with secukinumab after failure with ustekinumab. Four patients had primary failure with ustekinumab and 2 patients experienced secondary failure. After 12 weeks, 4 patients achieved PASI 90 with secukinumab [1].

Another study of 235 patients randomized 3 groups of anti-TNF nonresponders to treatment with secukinumab at
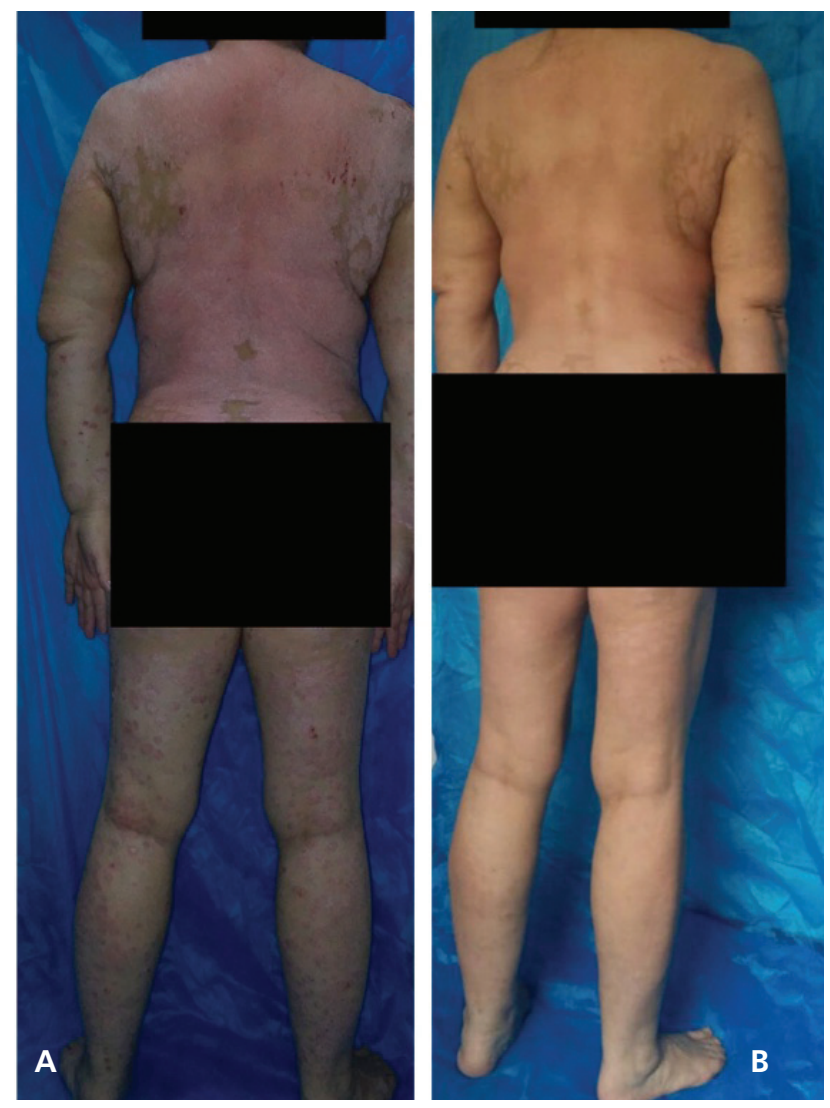

Figure 3. Clinical presentation. (A) After adalimumab treatment. (B) After 5 weeks of secukinumab treatment.

300 or $150 \mathrm{mg}$. The 3 groups were: patients with primary failure to anti-TNF $\alpha$; patients with secondary failure to anti-TNF $\alpha$; and patients who failed with more than one anti-TNF $\alpha$. The patients in the 3 groups achieved statistically significant rates of PASI 75 using secukinumab $300 \mathrm{mg}$ [2].

Magnano et al reported on 16 psoriatic patients previously treated with more than 1 systemic or biologic agent, without control of skin lesions. These patients were treated with secukinumab, and 8 of them obtained a complete clearance (PASI 100), 6 patients presented PASI 90, and 2 patients showed PASI 75. All patients presented improvement in the DLQI [3]. In addition, a case of successful treatment with secukinumab in recalcitrant psoriatic arthritis treated previously with 2 anti-TNF drugs was reported [4].

The case reported here was exposed to multiple systemic treatments resulting in inadequate disease control and adverse events that led to discontinuation of therapies. She failed to respond to both anti-TNF $\alpha$ and anti-IL12/23 drugs but reached PASI 100 in 8 weeks with secukinumab without any adverse effects. This indicates that each patient may have a specific behavior in the disease pathway and might respond better to certain drugs. Further studies are needed to determine which factors are essential to define the biologic of choice. 


\section{References}

1. Morgado-Carrasco D, Riera-Monroig J, Fustà-Novell X, Alsina Gibert M. Response to secukinumab after treatment failure with ustekinumab in 6 patients with plaque psoriasis. Actas Dermosifiliogr. 2018;109(6):565-567. DOI: 10.1016/j. ad.2017.07.019. PMID: 29169562.

2. Warren RB, Barker J, Burden AD, et al. Secukinumab has demonstrated efficacy and safety in hard-to-treat anti-tumor necrosis factor $\alpha$ failure patients from the United Kingdom and Republic of Ireland: Results of the SIGNATURE study. J Am
Acad Dermatol. 2018;79(3 Suppl 1): AB256. Poster Abstract. DOI: 10.1016/j.jaad.2018.05.1018.

3. Magnano M, Loi C, Patrizi A, et al. Secukinumab in multifailure psoriatic patients: the last hope? J Dermatolog, Treat. 2018;29(6):583-585. DOI: 10.1080/09546634.2018.1427206. PMID: 29334270.

4. Pelechas E, Memi T, Voulgari PV, Drosos AA. A case of recalcitrant psoriatic arthritis to tnf inhibitors improved after administration of secukinumab, an IL-17A inhibitor. Rheumatol Ther. 2017;4(2):509-513. DOI: 10.1007/s40744-017-0084-0. PMID: 29022197. 